\title{
Spatial Resolution of Domain Copying in a Magnetic Domain Expansion Readout Disk
}

\author{
P. W. M. Blom, J. J. L. Horikx, P. J. H. Bloemen, C. A. Verschuren, H. W. van Kesteren, H. Awano, and N. Ohta
}

\begin{abstract}
In a magnetic amplifying magnetooptical system (MAMMOS) disk, the copying process of a magnetic domain from the recording layer into the readout layer is investigated by using a laser spot diameter of $0.66 \mu \mathrm{m}$. Interference between neighboring bits occurs when the spatial resolution of the copy process is larger than half the domain size. It is observed that a reduction of the readout power from 2.3 to $2.0 \mathrm{~mW}$ corresponds to a decrease of the copy window from 0.5 to $0.06 \mu \mathrm{m}$. As a result, at $2.0 \mathrm{~mW}$ packed magnetic domains with a width of only $0.11 \mu \mathrm{m}$ have been resolved without interference by using domain expansion.
\end{abstract}

Index Terms-Domain expansion, magnetooptical recording, super resolution readout.

\section{INTRODUCTION}

$\mathbf{I}$ $\mathrm{N}$ AN optical recording system, the maximum achievable information density on a disk is limited by the size of the optical spot. Improvement of the recording density has mainly been focused on the size reduction of the diffraction limited optical spot. By using a blue laser diode in combination with high numerical aperture NA $=0.85$ focusing optics recording densities of $20 \mathrm{~GB}$ on a 12-cm optical disk have been achieved [1]. In magnetooptical systems using the writing strategy of laserpumped magnetic field modulation (LP-MFM), bits as small as one tenth of the laser spot size can be written [2]. Domains smaller than the optical resolution can be detected in readout by using magnetically induced super resolution (MSR) [3]. In this technique, the temperature distribution of the laser spot creates an aperture in an additional readout layer, and the domains are reproduced through the aperture as isolated bits. However, for very small domains, the MSR signals become too weak for appropriate readout. The recently introduced magnetic amplifying magnetooptical system (MAMMOS) technique [2] allows for both high resolution and large signals. The readout process of the MAMMOS technique can be divided into three parts: First, a recorded domain is copied from the recording layer into the readout layer by an increase of the magnetostatic coupling between the two layers due to heating with the laser beam. By simultaneously applying a magnetic readout field, the copied domain is then expanded in the readout layer. Finally, by applying an opposite field, the expanded domain is collapsed. Due to the domain expansion, a large signal is obtained for each recorded domain. After the first report on the

Manuscript received September 14, 2000; revised January 30, 2001.

P. W. M. Blom, J. J. L. Horikx, P. J. H. Bloemen, C. A. Verschuren, and H. W. van Kesteren are with Philips Research Laboratories, 5656 AA Eindhoven, The Netherlands.

H. Awano and N. Ohta are with Tsukuba Research Laboratory, Hitachi-Maxell Ltd., Ibaraki 300-2496, Japan.

Publisher Item Identifier S 0018-9464(01)07987-0.
MAMMOS technique [2], various types of MAMMOS with different media structures have been proposed, including the use of an in-plane magnetized readout layer [4] or an additional gating layer [5]. Another technique to increase readout signals, which is based on exchange coupled layers, is the domain wall displacement detection (DWDD) [6], in which the domain wall moves to a higher temperature region of the readout layer during illumination with a laser beam. Using the MFMMAMMOS readout method, a resolution for isolated magnetic domains of $0.04 \mu \mathrm{m}$ has been obtained empirically, with a minimum spacing between magnetic domains of $0.14 \mu \mathrm{m}$ [7]. Furthermore, packed magnetic domains that consist of $0.1-\mu \mathrm{m}$ domains with $0.1-\mu \mathrm{m}$ spaces could also be resolved [8]. In the present study, we investigate the spatial resolution of the copy process, i.e., the copy window size, as a function of the laser read power. The process of copying domains from the recording into the readout layer is characterized by a sharp threshold in read power. From the copied signals of $0.5-\mu \mathrm{m}$ domains, the width of the copy window has been obtained directly at various read powers. Under optimized readout conditions, MAMMOS signals from $0.11-\mu \mathrm{m}$ packed domains could be resolved.

\section{RESULTS}

The layer structure of the MAMMOS disk is $\mathrm{Si}_{3} \mathrm{~N}_{4}(60$ $\mathrm{nm}) / \mathrm{GdFeCo}(20 \mathrm{~nm}) / \mathrm{Si}_{3} \mathrm{~N}_{4}(5 \mathrm{~nm}) / \mathrm{TbFeCo}(50 \mathrm{~nm}) / \mathrm{Si}_{3} \mathrm{~N}_{4}$ $(20 \mathrm{~nm}) / \mathrm{Al}$ alloy $(30 \mathrm{~nm})$. The Curie temperature $\left(T_{c}\right)$ and compensation temperature $\left(T_{\text {comp }}\right)$ of the $\mathrm{GdFeCo}$ layer are $350{ }^{\circ} \mathrm{C}$ and $60{ }^{\circ} \mathrm{C}$, respectively. The $T_{c}$ and $T_{\text {comp }}$ for the TbFeCo layer are $270{ }^{\circ} \mathrm{C}$ and $20{ }^{\circ} \mathrm{C}$, respectively. The magnetic layers have been prepared by a magnetron sputtering process. The wavelength of the dynamic tester is $658 \mathrm{~nm}$ and the NA of the objective lens is 0.6 , resulting in a full-width at half-maximum (FWHM) diameter of the laser spot of $0.66 \mu \mathrm{m}$. In Fig. 1, the amplitude of the detected MO signal is shown as a function of the magnetic domain length. For domains smaller than $0.33 \mu \mathrm{m}$, which is half the laser spot size, it is evident that no modulation of the MO signal could be detected, as is indicated in the inset of Fig. 1. In order to study the copy process in the MAMMOS disk, we recorded domains with a width of $0.5 \mu \mathrm{m}$ using LP-MFM. Since these $0.5-\mu \mathrm{m}$ domains are well within the resolution of our tester, they can be observed without the use of domain expansion. From Fig. 1, it appears that the signal amplitude of these $0.5-\mu \mathrm{m}$ domains is typically half of the saturated value.

In Fig. 2, the signal amplitude of the $0.5-\mu \mathrm{m}$ domains, which are copied from the recording layer into the readout layer, is shown as a function of read power $P_{r}$ at a linear velocity of $1 \mathrm{~m} / \mathrm{s}$. We observe that at this velocity the copy process 


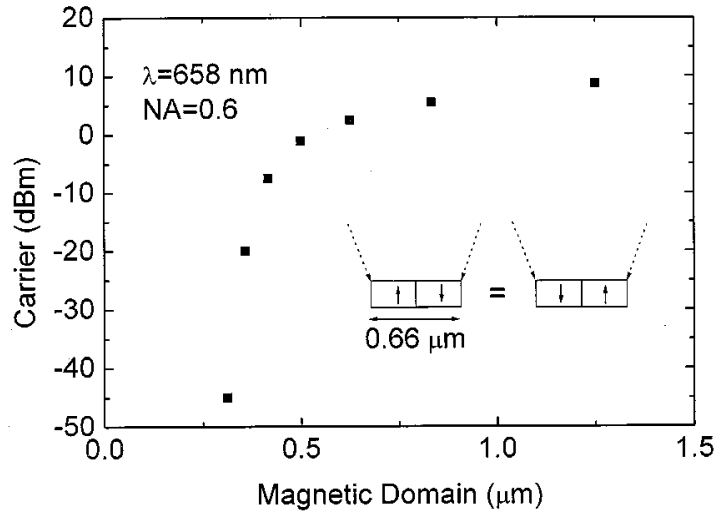

Fig. 1. Strength of a magnetic carrier as a function of magnetic domain width The laser wavelength $\lambda=658 \mathrm{~nm}$ combined with a numerical aperture (NA) of 0.6 corresponds to a spot diameter (FWHM) of $0.6 \lambda / \mathrm{NA}=0.66 \mu \mathrm{m}$. For domains $\leq 0.33 \mu \mathrm{m}$, as indicated in the inset, the MO signal is identical and the magnetic domains cannot be resolved.

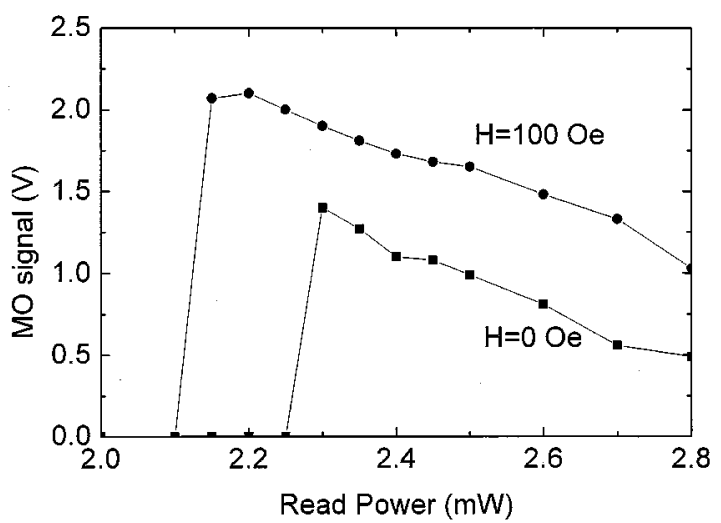

Fig. 2. MO signal of $0.5 \mu \mathrm{m}$ packed domains as a function of the read power. For a read power $P_{r}<2.25 \mathrm{~mW}(\mathrm{H}=\mathrm{Oe})$, the recorded bits are not copied into the readout layer. At $P_{r}=2.25 \mathrm{~mW}$, the magnetization of a selected domain in the recording layer is sufficiently enhanced by the temperature increase that the domain is copied into the readout layer, of which the coercive field $H_{c}$ has decreased by the temperature rise. When the magnetization of a domain is enhanced by an external magnetic field, the copy threshold shifts to lower read powers; a field of 100 Oe corresponds to a shift of $0.15 \mathrm{~mW}$.

has a sharp threshold at $P_{r}=2.25 \mathrm{~mW}$. For the TbFeCo recording layer and $\mathrm{GdFeCo}$ readout layer, the compensation temperatures are typically around room temperature [8]. As a result, with increasing temperature the magnetization of both layers increases, whereas simultaneously the coercive field of the GdFeCo readout layer drops [8]. At $P_{r}=2.25 \mathrm{~mW}$, the magnetization and stray field of the $\mathrm{TbFeCo}$ recording layer have sufficiently increased to reverse the magnetization of the $\mathrm{GdFeCo}$ readout layer. When a magnetic field is applied with a direction such that it enhances the stray field of the recording layer, the copy threshold shifts to lower readout powers, as also shown in Fig. 2. For a field of 100 Oe, the shift typically amounts to $0.15 \mathrm{~mW}$. In the MAMMOS detection technique, a readout field is applied and the magnetic domains will be copied into the readout layers at read powers slightly below the zero-field threshold. The copy threshold at zero magnetic field is also dependent on the linear velocity $v$ of the disk. At $v=10$ $\mathrm{m} / \mathrm{s}$, a read power of $4.5 \mathrm{~mW}$ is required to copy the $0.5-\mu \mathrm{m}$ domains into the readout layer. For very small domains, it is evident that the spatial resolution of the copy process should
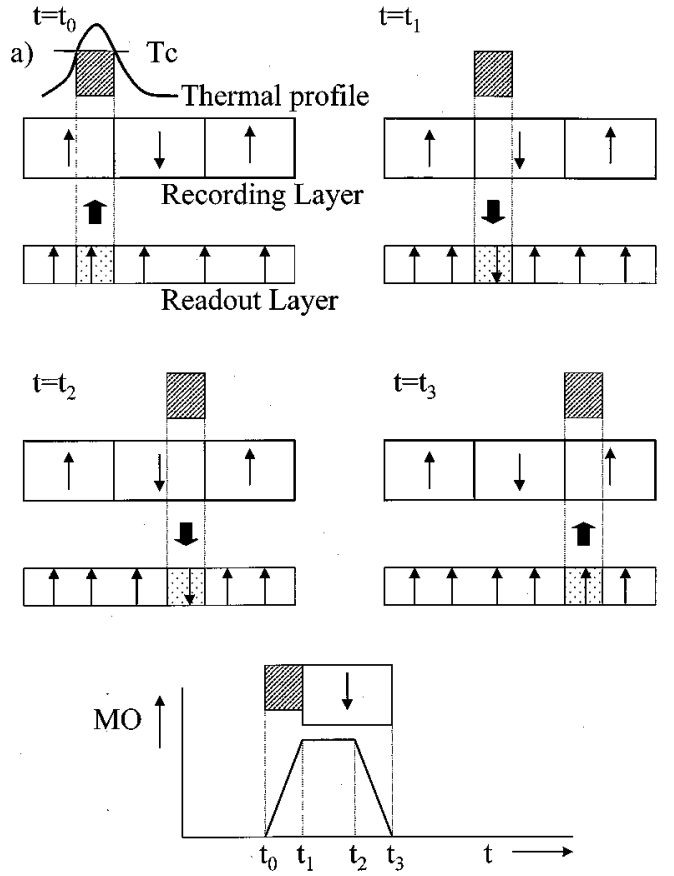

b)
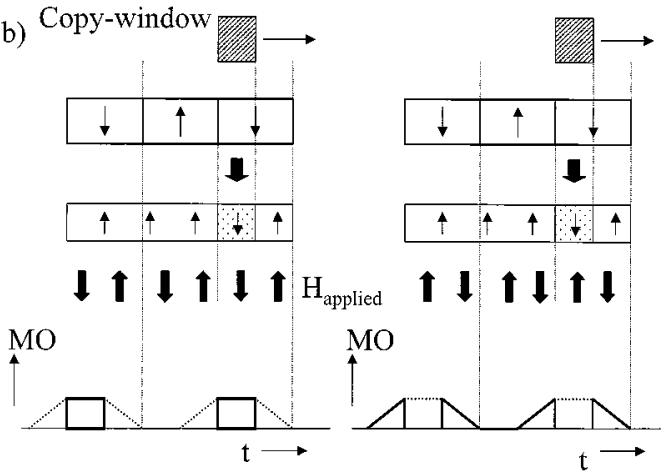

Fig. 3. Schematic representation of the copy process in a MAMMOS disk. In (a) the thermal profile as a result of the laser spot is indicated. Only in a certain region of the profile the temperature is sufficient to enable the copy process, as indicated by the hatched area. At time $t=t_{0}$ the copy window has reached a magnetic mark with magnetization opposite to the readout layer. At $t=t_{1}$ the copy window is completely inside the mark and the MO signal reaches its maximum value until $t=t_{2}$. For $t>t_{2}$, the copy window moves out of the mark and the MO signal decreases until it reaches zero at $t=t_{3}$. The time-interval $t_{3}-t_{0}$ is translated via the linear velocity into a total width of the MO signal, which corresponds to the sum of the length of the copied domain and the copy window. In (b) the effect of an externally applied modulated magnetic field, which is characteristic for MAMMOS detection, is indicated. For read powers which are equal or smaller than the copy threshold application of a field in opposite direction to the magnetization of the recording layer suppresses the copy process. On the left scheme, the magnetic field is in phase with the recorded data and the part of the MO signal that is suppressed by the applied field (block arrows) is indicated by the dashed line. On the right scheme, the applied field is out of phase with the data.

be high enough to select and copy only one bit of the recording layer into the readout layer. In the reports on MAMMOS detection so far, the width of the area that is copied, the spatial copy window, has not been addressed.

In order to determine the width of the copy window in the MAMMOS disk, we first consider the MO signal of domains that are copied into the readout layer at zero-field condition, as schematically indicated in Fig. 3. In Fig. 3(a), the thermal profile of the laser spot is indicated together with the recording 
layer and the readout layer, which is uniformly magnetized. Only in that part of the profile, where the temperature is sufficiently high to initiate the copy process, is the information of the recording layer copied into the readout layer; the width of this copy window is indicated by the shaded area. Copying a domain of the recording layer with the same magnetization as the readout layer, called a nonmark, does not give rise to an abrupt change of the magnetization in the readout layer: as a result, no signal peak is observed. When the copy window reaches the magnetic domain with magnetization opposite to the readout layer $\left(t=t_{0}\right)$, which is called a mark, the MO signal will start to increase. At $t=t_{1}$, the copy window is completely inside the mark and the MO signal reaches its maximum value. At $t=t_{2}$, the front of the window has reached the end of the mark, and from there the MO signal starts to decrease. Finally, at $t=t_{3}$, the copy window is outside the mark. Since the copy process of a "nonmark" does not change the magnetization of the readout layer, the MO signal is zero again. From Fig. 3(a), it is clear that the total width of the MO signal from one mark corresponds to the sum of the domain length and the width of the copy window. Thus, from the MO signal of a copied domain, the width of the copy window can directly be derived.

For the MAMMOS detection technique, it is relevant to determine the copy window at read powers below the zero-field copy threshold. In order to make the recorded domains visible below the copy threshold, an external magnetic field with the right direction needs to be applied, as shown in Fig. 2. In order to mimic the conditions for MAMMOS readout, an alternating magnetic readout field with a frequency of twice the data frequency is applied. In Fig. 3(b), the effect of such an additional alternating readout field (block arrows) is indicated: For read powers below the copy threshold, the $0.5-\mu \mathrm{m}$ domains are only copied when the field has the correct direction (solid line). For the other field direction, the copy process is blocked; the resulting MO signal is suppressed whenever the direction of the readout field is opposite to the magnetization of the mark (dotted line). In Fig. 3(b), the copied domains are shown for the case that the applied field is in phase with the data (left) or out of phase (right).

In order to measure the envelope of the copied magnetic mark of $0.5 \mu \mathrm{m}$, a magnetic readout field of typically 100 Oe with a period of $0.5 \mathrm{MHz}$ is applied, as shown in the upper part of Fig. 4. For domain expansion, fields larger than 150 Oe are required. For a read power of $2.30 \mathrm{~mW}$, which is slightly above the copy threshold, a mark is only copied into the readout layer when the readout field has the correct direction. Since the data frequency amounts to $1.0 \mathrm{MHz}(v=1 \mathrm{~m} / \mathrm{s})$, the magnetic readout field assists the copy process during the passage of the copy window through a mark, and disables the copy process during the passage of the next mark. As a result, the envelope of a copied mark (dashed line) is obtained without interference from a neighboring mark. The dotted vertical lines represent a distance of $1 \mu \mathrm{m}$. It is shown that the width of the MO signal at $P_{r}=2.30 \mathrm{~mW}$ is equal to $1 \mu \mathrm{m}$, from which a copy window of $0.5 \mu \mathrm{m}$ is obtained. In order to mimic MAMMOS readout conditions, we have also applied a readout field of 100 Oe with a frequency of $2 \mathrm{MHz}$. As expected from Fig. 3(b), the envelope is now only partially visible due to the switching of the magnetic field during the passage of the mark. At $P_{r}=2.15$

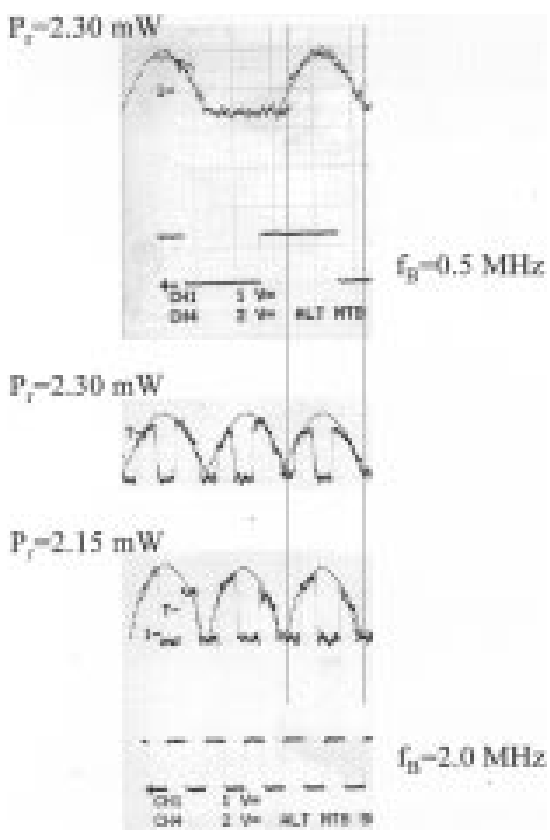

Fig. 4. MO signal of $0.5-\mu \mathrm{m}$ domains in the presence of an alternating magnetic field; upper part: $100 \mathrm{Oe}, 0.5 \mathrm{MHz}$; lower part: $100 \mathrm{Oe}, 2 \mathrm{MHz}$. The linear velocity of the disk amounts to $1 \mathrm{~m} / \mathrm{s}$. The dashed lines correspond to the envelope of the copied domains. The copy window decreases from 0.5 to 0.23 $\mu \mathrm{m}$ when the read power is reduced from 2.3 to $2.15 \mathrm{~mW}$, respectively.

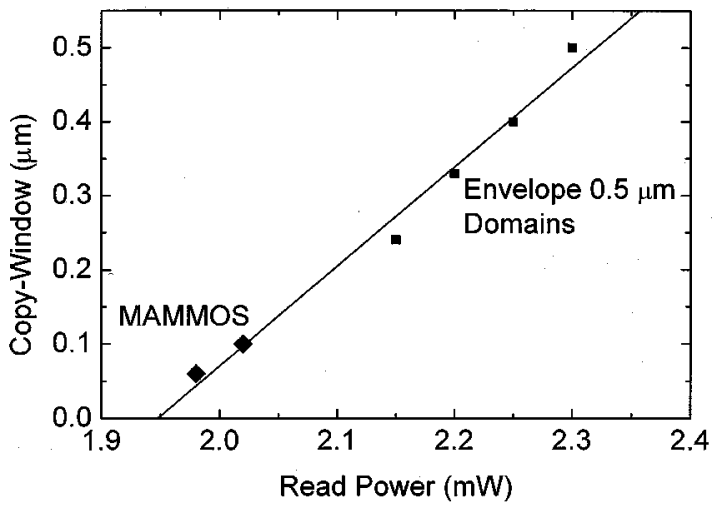

Fig. 5. Copy window versus read power as obtained from the envelope of the copied $0.5-\mu \mathrm{m}$ domains (squares). Furthermore, the copy windows around 2 $\mathrm{mW}$ as estimated from MAMMOS measurements are included (diamonds).

$\mathrm{mW}$ (lower part of Fig. 4), the width of the MO signal is significantly smaller and amounts to $0.73 \mu \mathrm{m}$, which for a magnetic domain of $0.5 \mu \mathrm{m}$ results in a copy window of about 0.23 $\mu \mathrm{m}$. In Fig. 5, the measured spatial width of the copy window, as obtained from the envelope signal of the $0.5-\mu \mathrm{m}$ domains, is shown as a function of read power (squares). For $P_{r}$ smaller than $2.15 \mathrm{~mW}$, the applied magnetic field, which is required to initiate the copy process, becomes larger than $150 \mathrm{Oe}$ and the copied domains directly expand. This requires a different analysis to determine the copy window, as explained below.

The most important question to address now is how small the copy window has to be in order to reproduce MAMMOS signals of packed domains without interference. In Fig. 6, the copied (middle) and expanded (bottom) MO signals are schematically indicated for a copy window with a width of half the magnetic domain size (left) and a width equal to the domain size 


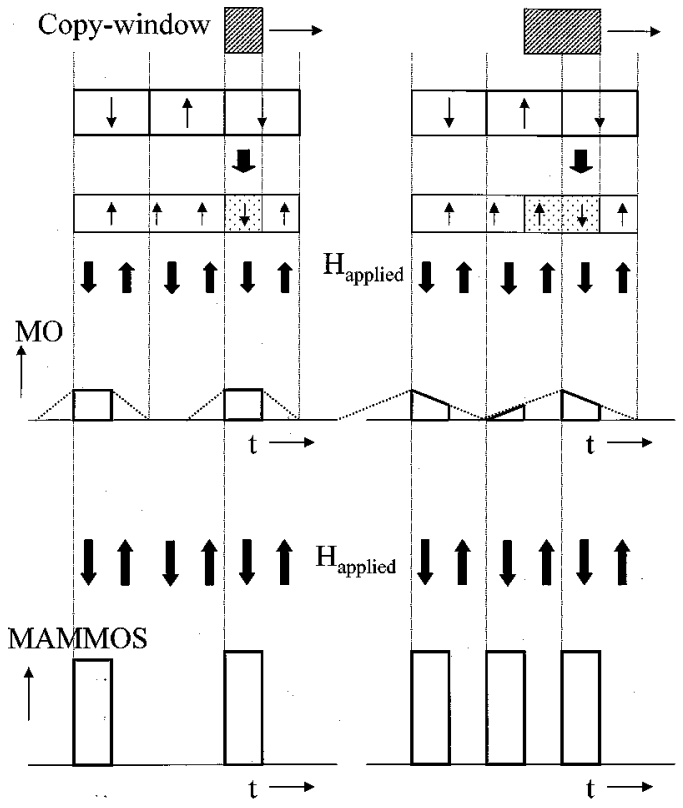

Fig. 6. Schematic representation of the copy process and domain expansion for a copy window with a width of half (left) and full (right) magnetic domain size. For a copy window smaller than half the domain size each mark in the recording layer (magnetization down) corresponds to a MAMMOS peak. For copy windows larger than half the domain size additional MAMMOS peaks at nonmark positions appear due to overlap of the copied domains.

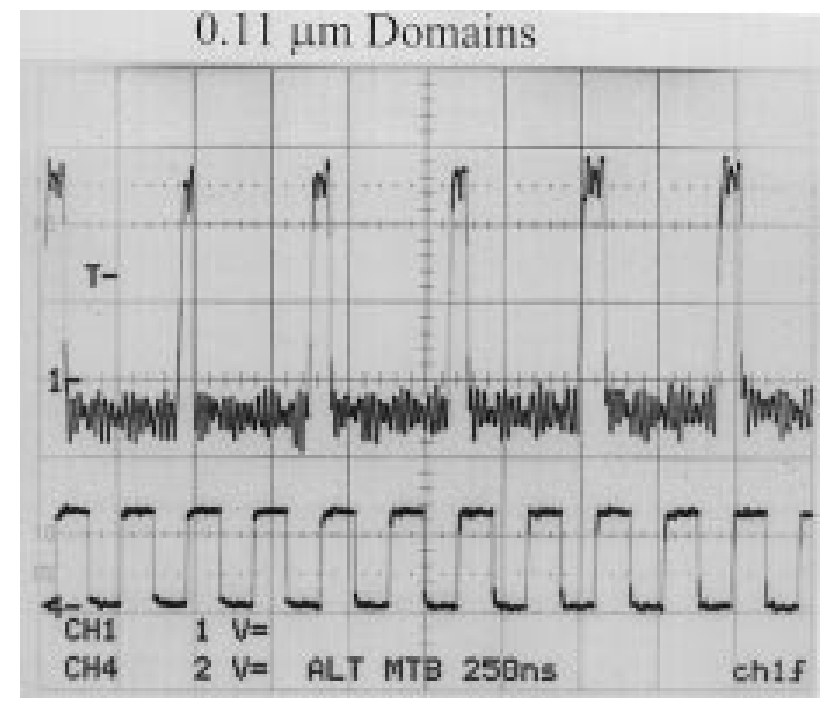

Fig. 7. MAMMOS signal of $0.11-\mu \mathrm{m}$ packed domains using a read power of $1.83 \mathrm{~mW}$ at a linear velocity of $0.5 \mathrm{~m} / \mathrm{s}$. The $0.11-\mu \mathrm{m}$ domains can be resolved without interference between neighboring marks.

(right). It appears that copy windows larger than half the domain size give rise to additional MAMMOS peaks (from nonmarks), which of course is unwanted. Thus, from Fig. 5, it appears that for read powers below $2.15 \mathrm{~mW}$, the copy window is smaller than half the magnetic domain size of the recorded domains of $0.5 \mu \mathrm{m}$. This is in agreement with the experimental observation that for $P_{r}=2.10 \mathrm{~mW}$ and below MAMMOS signals of the $0.5 \mu \mathrm{m}$ packed domains are observed without interference. In order to increase the resolution toward smaller bits, it is clear from Fig. 5 that a further reduction of the read power is required.
For 0.2- $\mu \mathrm{m}$ domains, proper MAMMOS signals were observed for read powers below $2.02 \mathrm{~mW}$. Read powers larger than 2.02 $\mathrm{mW}$ gave rise to additional peaks. Thus, at $2.02 \mathrm{~mW}$, the copy window is nearly equal to $0.1 \mu \mathrm{m}$, which is half the domain size. Likewise, for $0.12-\mu \mathrm{m}$ bits, a maximum read power of $1.98 \mathrm{~mW}$ has been obtained, which then corresponds to a copy window of $0.06 \mu \mathrm{m}$. These data are also included in Fig. 5 (diamonds), and we observe that they nicely fit the apparent linear behavior of copy window versus read power, as already obtained from the envelope of the $0.5-\mu \mathrm{m}$ domains. Finally, as shown in Fig. 7, MAMMOS peaks of packed domains with a width of only 0.11 $\mu \mathrm{m}$ could be resolved. Thus, according to these results, the minimum separation between marks in order to avoid interference should be close to $0.1 \mu \mathrm{m}$. This is in agreement with the value of $0.1 \mu \mathrm{m}$, as reported by Awano et al. [8].

\section{CONCLUSION}

Our experiments demonstrate that the maximum attainable resolution for packed domains in the MAMMOS readout technique is limited by the spatial width of the copy window. However, it should be noted that the readout power cannot be continuously reduced in order to further increase the resolution. A reduction of readout power reduces the magnetization of the recorded bits; as a result, the external readout field that compensates this effect in the copy process has to become larger. If the external field, which is required to copy the domain, becomes larger than the coercive field of the readout layer, the MO signals will just follow the applied field. The maximum attainable resolution is thus a complex interplay between the temperature dependence of the magnetization of the recording layer, the coercive field of the readout layer, and the coupling between the layers. Knowledge of the spatial resolution of the copy process will be a valuable tool for further optimization of the resolution of the MAMMOS technique.

In conclusion, we have investigated the spatial copy window in a MAMMOS readout disk. In order to resolve recorded domains without interference, the copy window should be equal to or smaller than half the domain size. It is observed that a reduction of the read power from 2.30 to $1.98 \mathrm{~mW}$ gives rise to a decrease of the copy window from $0.5 \mu \mathrm{m}$ toward $0.06 \mu \mathrm{m}$. Knowledge of the read power dependence of the copy window directly provides the maximum read power, which can be used to resolve domains of a given width using MAMMOS.

\section{REFERENCES}

[1] T. Narahara, S. Kobayashi, M. Hattori, Y. Shimpuku, G. van den Enden, J. A. H. M. Kahlman, M. van Dijk, and R. van Woudenberg, "Optical disc system for digital video recording," Jpn. J. Appl. Phys., pt. 1, vol. 39, no. 2B, p. $912,2000$.

[2] H. Awano, S. Ohnuki, H. Shirai, and N. Ohta, "Magnetic domain expansion readout for amplification of an ultra high density magneto-optical recording signal," Appl. Phys. Lett., vol. 69, p. 4257, 1996.

[3] K. Aratani, A. Fukumoto, M. Ohta, M. Kaneko, and K. Watanabe, "Magnetically induced super resolution in novel magneto-optical disk," in Proc. SPIE Optical Data Storage 1991, vol. 1499, 1991, p. 209.

[4] X. Ying, K. Shimazaki, H. Awano, M. Yoshihiro, H. Watanabe, N. Ohta, and K. V. Rao, "Magnetic expansion readout of $0.2 \mu \mathrm{m}$ packed domains on a magneto-optical disk with an in-plane magnetized readout layer," Appl. Phys. Lett., vol. 72, p. 614, 1998. 
[5] H. Awano, H. Shirai, H. Watanabe, K. Shimazaki, and N. Ohta, "Signal enhancement of $0.2 \mu \mathrm{m}$ packed domain in magnetic domain expansion readout disk with gating layer," IEEE Trans. Magn., vol. 34, p. 2003, 1998.

[6] T. Shiratori, E. Fujii, Y. Miyaoka, and Y. Hozumi, "High-density magneto-optical recording with domain wall displacement detection," J. Magn. Soc. Jpn. (Proc. Magneto-Optical Recording Int. Symp., Yamagata 1997), vol. 22, no. S2, p. 47, 1998.
[7] N. Takagi, K. Mitana, H. Awano, K. Shimazaki, and N. Ohta, "MAMMOS readout with magnetic field modulation," J. Magn. Soc. Jpn. (Proc. Magneto-Optical Int. Symp. 1999), vol. 23, no. S1, p. 161, 1999.

[8] H. Awano, M. Sekine, M. Tani, H. Ido, M. Yoshihiro, K. Shimazaki, and N. Ohta, "Domain dynamic phenomena of domain expansion readout disk," J. Magn. Soc. Jpn. (Proc. Magneto-Optical Int. Symp. 1999), vol. 23 , no. S1, p. $147,1999$. 